\title{
Racial/Ethnic Representation Among American Board of Family Medicine Certification Candidates from 1970 to 2020
}

\author{
Ting Wang, PhD, Thomas R. O'Neill, PhD, Warren P. Newton, MD, MPH, \\ Kennedi Hall, MPH, and Aimee R. Eden, PhD, MPH
}

Background: Because improved patient outcomes and experiences have been associated with health care workforce diversity, efforts to create a diverse family physician workforce have increased. However, a metric that could properly measure family physician representation in various contexts has seldom been studied.

Objective: The goal of this study is to propose a new metric $\operatorname{logRQ}$ and use it to examine the diversification progress of American Board of Family Medicine (ABFM) certification candidates relative to national, state, and historic populations, as well as medical school matriculants.

Methods: We obtained race/ethnicity for certification candidates from the 2014 to 2020 ABFM Certification Examination Registration questionnaire and examined racial/ethnic representation relative to various populations via logRQs.

Results: The total sample comprised 26,368 initial certification candidates and 55,347 continuing certification candidates. Asian, Hispanic, and Black's $\operatorname{logRQ}$ increased by $0.51,0.42$, and 0.41 , respectively, in initial certification candidates compared with continuing certification candidates. In addition, $\operatorname{logRQ}$ standard deviation ranged from 0.19 to 0.87 across States, indicating state-level variation. Although Black and Hispanic remained underrepresented, the degree of underrepresentation had improved substantially across the past 5 decades, with $\operatorname{logRQ}$ increasing from -2.12 (Black) and -1.16 (Hispanic) in the $1970 \mathrm{~s}$ to -0.46 (Black) and -0.68 (Hispanic) in the 2010s. The race/ethnicity logRQs of 2020 initial certification candidates relative to 2013 to 2014 medical school matriculants were all near 0 , reflecting equitable representation.

Conclusion: We utilized the proposed metric $\operatorname{logRQ}$ to quantify the advancement in representation among ABFM certification candidates in different contexts. The proposed $\operatorname{logRQ}$ may serve as a useful tool to monitor representation progress systematically. (J Am Board Fam Med 2022;35:9-17.)

Keywords: Certification, Ethnic Groups, Minority Groups, Family Medicine, Family Physicians, Primary Health Care, Surveys and Questionnaires, Workforce

\section{Introduction}

There is a growing body of evidence that suggests racial and ethnic concordance between the physician and patient is related to better physician-patient communication, lower infant mortality, improved

This article was externally peer reviewed.

Submitted 30 July 2021; revised 7 October 2021; accepted 8 October 2021.

From the American Board of Family Medicine, Lexington, KY (TW, TON, WPN, KH, ARE).

Funding: The authors received no funding to conduct this research.

Conflict of interest: None.

Corresponding author: Ting Wang, PhD, 1648 McGrathiana Pkwy, Ste 550, Lexington, KY 40511 (E-mail: twang@ theabfm.org). health care use, lower health care costs, and higher patient compliance and satisfaction. ${ }^{1-6}$ Over the last decades, there have been sustained efforts to diversify the physician workforce. ${ }^{7}$ While family medicine is less diverse than some other primary care specialties, ${ }^{8}$ the family physician workforce has become more diverse over time; from 1987 to 2017, the percentage of Black or African American initial certifiers increased from $1.3 \%$ to $7.8 \%$ and Hispanic or Latino initial certifiers increased from $2.3 \%$ to $9.1 \%{ }^{9}$ However, the reason for this increase in the percentage of Black or African American and Hispanic or Latino physicians is unclear: it may be simply due to the national population demographic composition change over the past decades or 
diversification among medical school matriculants. In addition, ABFM candidate population is composed of both initial certification candidates and continuing certification candidates, yet the extent to which the racial/ethnic representation across initial certification candidates and continuing certification candidates relative to the national population has not been examined in detail. Likewise, no previous research has examined whether representation varies at the state level. In seeking statistically sound methods to measure these diversification processes systematically, we propose a new metric.

The new metric proposed in this study is an extension of the representation quotient (RQ) utilized by Lett et al, ${ }^{10}$ and the rank equity index (REI) proposed by Fassiotto. ${ }^{11}$ In addition to taking the ratio between two proportions as RQ and REI, we further take the $\log$ of the ratio. This extension has several methodological advantages. First, it transforms the range of the metric from positive only to both positive and negative, facilitating the use of linear regression model by allowing the outcome variable to support the range from $-\infty$ to $+\infty$. $^{12}$ Second, $\log \mathrm{RQ}$ maps the quantity into a symmetric and continuous scale rather than the nonsymmetric scale represented by quotient. Specifically, in the RQ or REI metric, underrepresentation is in the range $(0,1)$, whereas the overrepresented range is $(1,+\infty)$. This nonsymmetric range leads to indistinguishable differences when comparing the extent of underrepresentation and large differences in overrepresentation comparison. ${ }^{10}$ In contrast, the magnitude of $\log R Q$ reflects the extent of the overrepresentation and underrepresentation symmetrically. For example, logRQ of 1.38 indicates 4 times $(1.38=\log (4))$ of overrepresentation, and $\log R Q$ of -1.38 indicates 4 times of underrepresentation $(-1.38=\log (1 / 4))$. The equitable representation is reflected in $0(0=\log (1))$, which has a somewhat intuitive interpretation. This $\log R Q$ metric can be utilized in any proportion ratio context. In this study, we will apply this metric to examine the diversification progress of ABFM certification candidates relative to national, state, and historic populations, as well as medical school matriculants.

\section{Methods}

\section{Data Sources}

We obtained self-reported race and ethnicity data from the demographics section of the application to sit for the ABFM certification examination from
2014 to 2020. Before 2014, the ABFM did not collect race/ethnicity information from initial certification candidates. The national and state-level race/ ethnicity information was obtained from the 2019 Census Bureau's American Community Survey (ACS). To be consistent with the ACS race and ethnicity categorization, race and ethnicity were considered separately. Specifically, ethnicity was dichotomized as Hispanic or Latino and NonHispanic; race was categorized as Asian, White, Black or African American, American Indian/Alaska Native, Native Hawaiian/other Pacific Islander, or Other (the Other category was added as a response option in 2017). On the national level analysis, the numerators of the $\log \mathrm{RQs}$ were the race/ethnicity proportion in initial and continuing certification candidates, respectively. The denominators were the national race/ethnicity proportion.

Participants' primary practice state was also obtained from the ABFM database. For participants with primary practice addresses in more than one State, we used the most recent one to reflect updated workforce distribution. On the state level analysis, the numerator of the $\log \mathrm{RQ}$ was the race/ ethnicity proportion in initial certification candidates, since they represent the future family medicine physician workforce; and the denominator was the corresponding state race/ethnicity proportion.

The historic national race/ethnicity composition from 1970 (the earliest initial certification attempt in the sample) to 2010 was retrieved from Wikipedia website, ${ }^{13}$ which extracts information from historic Census Bureau briefs that were no longer available on line. These compositions were typically updated every decade. However, the only consistent race/ethnicity categories across the previous 5 decades (1970s to 2010s) and corresponding to $\mathrm{ABFM}$ race/ethnicity categories were White, Black or African American, Asian, American Indian or Alaska Native, Hispanic or Latino. Hence, the $\operatorname{logRQs}$ were only computed for these categories across decades. The numerator for the $\log \mathrm{R}$ was the race/ethnicity proportion of family physicians who made the certification attempt within a particular decade; the denominator was the corresponding decade race/ethnicity populational proportion.

Medical school matriculants' race/ethnicity was obtained from Association of American Medical Colleges (AAMC) website. ${ }^{14}$ Since AAMC changed the race/ethnicity categorization in 2012 and it typically takes 7 years (assuming regular progression) 
for medical school matriculants to be eligible for the initial certification examination, the only comparable cohort eligible for 2020 initial certification examination was the 2013 to 2014 medical school matriculant group. All race/ethnicity categories in the ABFM database exist in AAMC aggregated matriculants' data. The denominator of the logRQ in this context was the proportion of certain race/ethnicity of matriculants in 2013 to 2014 school year, serving as the entry-level of the workforce; the numerator was the proportion of the corresponding race/ethnicity physicians who made the certification attempts in 2020, representing a later stage in the educational pipeline.

\section{Statistical Analysis}

We contrasted the proportion of race/ethnic groups in initial and continuing certification candidates by conducting $\chi^{2}$ tests. The representation of initial and continuing certification candidates compared with the national population composition, States representation variation, initial certification candidates by decades relative to historic population composition, and medical school matriculants are all based on the proposed $\log \mathrm{R}$.
This study was approved by the institutional review board at the American Academy of Family Physicians. All statistical analyses were performed in $\mathrm{R}$ version 4.0.2 ( $\mathrm{R}$ Foundation for Statistical Computing, Austria).

\section{Results}

The sample included 26,368 initial certification candidates and 55,347 continuing certification candidates. Their demographic information regarding gender, location of medical training, medical degree type, and year of examination are shown in Table 1. Initial certification candidates had a higher percentage of female, Doctor of Osteopathic Medicine (DO) degree holders, and international medical school graduates compared with continuing certification candidates. The race and ethnicity proportions for initial and continuing certification candidates are shown in Table 2. The distributions of race and ethnicity differ significantly between initial certification candidates and continuing certification candidates (ethnicity: $\chi^{2}=264.13, d f=1$, $P<.001$; race: $\left.\chi^{2}=1784.2, d f=5, P<.001\right)$. Compared with continuing certification candidates, the

Table 1. Demographic Information of Initial and Continuing Certification Candidates

\begin{tabular}{lcc}
\hline & $\begin{array}{c}\text { Initial Certification Candidates } \\
(\mathrm{N}=26,368) \\
\mathrm{n}(\%)\end{array}$ & $\begin{array}{c}\text { Continuing Certification Candidates } \\
(\mathrm{N}=55,347) \\
\mathrm{n}(\%)\end{array}$ \\
\hline $\begin{array}{l}\text { Sex } \\
\text { Female }\end{array}$ & $\begin{array}{c}23010(41.6) \\
\text { Male }\end{array}$ & $32337(58.4)$ \\
Degree & $14319(54.3)$ & $50195(90.7)$ \\
MD & $12049(45.7)$ & $5152(9.3)$ \\
DO & & \\
Medical Training* & & \\
United States & \\
International & $20834(79.0)$ & $44860(81.5)$ \\
Year & $5534(21.0)$ & $10353(18.7)$ \\
2014 & & $10690(19.3)$ \\
2015 & $17572(66.5)$ & $8413(15.2)$ \\
2016 & $8796(33.4)$ & $8445(15.3)$ \\
2017 & & $8560(15.5)$ \\
2018 & $3939(14.9)$ & $7643(13.8)$ \\
2019 & $3606(13.7)$ & $8539(15.4)$ \\
2020 & $3604(13.7)$ & $3057(5.5)$ \\
\hline
\end{tabular}

Abbreviations: DO, Doctor of Osteopathic Medicine; MD, Doctor of Medicine.

${ }^{*}$ Candidates who graduated from Canadian medical school are categorized in U.S. training for training and performance similarities.

${ }^{\dagger}$ There were 134 continuing certification candidates without medical training country records, not shown in this table. 
Table 2. Race/Ethnicity Proportion and $\operatorname{logRQ}$ of Initial Certification and Continuing Certification Candidates, Relative to the National Population Race/Ethnicity Proportion

\begin{tabular}{|c|c|c|c|c|c|}
\hline & \multirow{2}{*}{$\begin{array}{c}\text { National } \\
\text { Population* } \\
\%\end{array}$} & \multicolumn{2}{|c|}{$\underline{\text { Initial Certification Candidates }}^{\dagger}$} & \multicolumn{2}{|c|}{ Continuing Certification Candidates $^{\ddagger}$} \\
\hline & & $\%$ & $\log R Q$ & $\%$ & $\log R Q$ \\
\hline Race & $\%$ & $\%$ & $\log \mathrm{RQ}$ & $\%$ & $\log \mathrm{RQ}$ \\
\hline White & 72.0 & 61.9 & -0.15 & 75.9 & 0.04 \\
\hline Black or African American & 12.8 & 8.2 & -0.45 & 5.4 & -0.84 \\
\hline Asian & 5.7 & 23.7 & 1.43 & 14.2 & 0.93 \\
\hline American Indian or Alaska Native & 0.9 & 0.9 & 0.00 & 0.8 & -0.12 \\
\hline Native Hawaiian or Other Pacific Islander & 0.2 & 0.5 & 0.92 & 0.6 & 0.92 \\
\hline Other & 5.0 & 4.9 & -0.02 & 3.1 & -0.42 \\
\hline \multicolumn{6}{|l|}{ Ethnicity } \\
\hline Hispanic or Latino & 18.4 & 9.3 & -0.68 & 6.2 & -1.07 \\
\hline
\end{tabular}

Abbreviation: RQ, representation quotient.

*National Population is from the 2019 Census Bureau (N = 328.2 Million).

${ }^{\dagger}$ Initial Certification Candidates are those that applied to ABFM from 2014 to 2020 ( $\mathrm{N}=26,368$ ).

${ }^{\ddagger}$ Continuing Certification Candidates are those that applied to ABFM from 2014 to 2020 (N = 55,347).

proportion of White initial certification candidates decreased by $14.0 \%$ (75.9\% vs $61.9 \%)$, while Asian, Hispanic or Latino, and Black or African American proportion increased by $9.5 \%, 3.1 \%$, and $2.8 \%$, respectively. The remaining racial groups, including American Indian or Alaska Native, Native Hawaiian or Other Pacific Islander and Other, have proportion changes less or equal to $1.8 \%$. In summary, the initial certification candidates are more diverse than continuing certification candidates. The increase in percentages is largest in Asian, followed by Hispanic or Latino and Black or African American.

As Table 2 shows, although initial certification candidates became more racially/ethnically diverse over this period (2014 to 2020), Black or African American and Hispanic or Latino were still underrepresented compared with the national population (Black or African American: $8.2 \%$ vs 12.8\%; Hispanic or Latino: $9.3 \%$ vs $18.4 \%$ ), yielding negative $\log \mathrm{RQ}$ as -0.45 and -0.68 , respectively. Conversely, Asian and Native Hawaiian or Other Pacific Islanders were overrepresented with positive $\log \mathrm{RQ}$ as 1.43 and 0.92 . White and American Indian or Alaska Native generally represented the national population with near-zero $\log \mathrm{RQs}(-0.15$ and 0.00 , respectively). The Other category was only available for 2017 and 2018 candidates, so the proportion of Other groups may not be comparable to the national proportion.

Regarding state-level $\log \mathrm{RQ}$ variation, Figure 1 shows $\log \mathrm{RQ}$ choropleth maps for initial certification candidates by race/ethnicity across all States. Blue shadings imply underrepresentation $(\log R Q<0)$, and orange shadings imply overrepresentation $(\log R Q>$ $0)$. States shown in gray had no candidates identifying with the corresponding race/ethnicity, yielding noncomputable $\log$ RQs. Asians were overrepresented in almost all States, with a state-level mean and standard deviation (SD) of $\log \mathrm{RQ}$ as $1.67(0.63)$, whereas Black or African Americans and Hispanic or Latinos were underrepresented in most States with $\log \mathrm{RQ}$ mean (SD) as $-0.2(0.75)$ and -0.51 (0.45), respectively. Whites were generally reflective of the state-level population proportion, with $\log \mathrm{RQ}$ as $-0.03(0.19)$. American Indians were overrepresented in the east but underrepresented in the west, as reflected in the orange and blue distribution in Figure 1. This pattern results in the largest SD in $\log R Q$ as 0.87 . Although Native Hawaiians/Other Pacific Islanders were overrepresented generally in States with computable logRQs, most were located near the coasts.

Table 3 displays the race/ethnicity proportion and $\log \mathrm{RQ}$ of initial certification candidates every decade since the 1970s, compared with populational race/ethnicity over the same periods. Although Black or African American and Hispanic or Latino were still underrepresented in the last 2 decades, the degree of underrepresentation had improved drastically across the past 5 decades, with $\operatorname{logRQ}$ increasing from -2.12 (Black or African American) and - 1.16 (Hispanic or Latino) in the 1970 s to -0.46 (Black or African 
Figure 1. Choropleth maps of $\operatorname{logRQ}$ for initial certification candidates by race/ethnicity and States. Note. A $\operatorname{logRQ}$ greater than 0 indicates that a group is overrepresented among family medicine initial certification candidates relative to the US population, and a $\operatorname{logRQ}$ less than 0 indicates that a group is underrepresented. The gray-colored States indicate no candidates identify with the corresponding race/ethnicity. Abbreviation: RQ, representation quotient.

Asian $\log R Q$

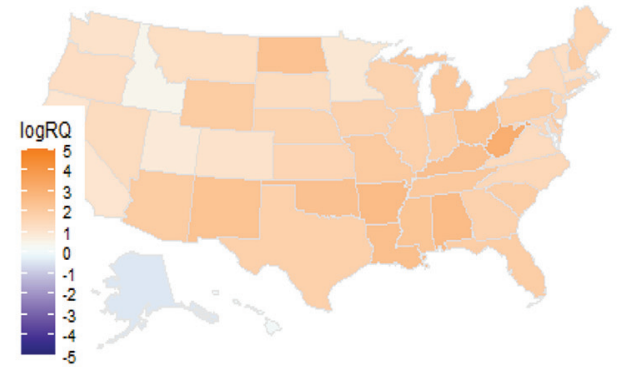

Black or African American $\log R Q$

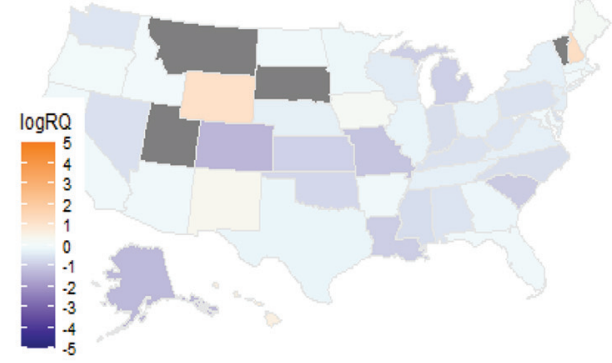

American Indian or Alaska Native logRQ

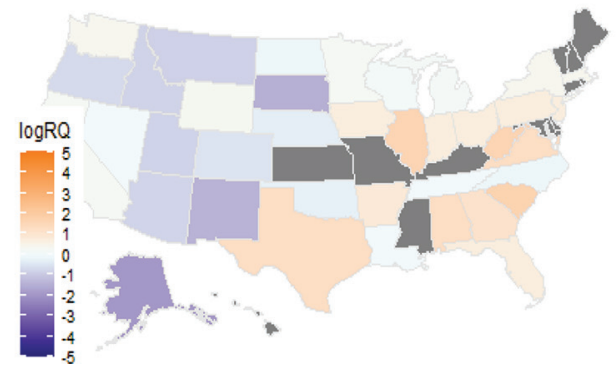

White $\log R Q$

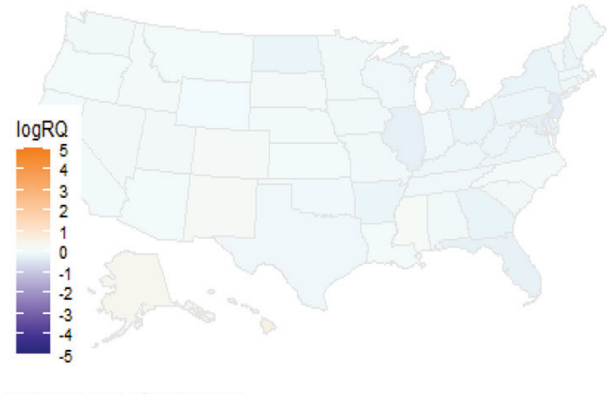

Hispanic or Latino $\log R Q$

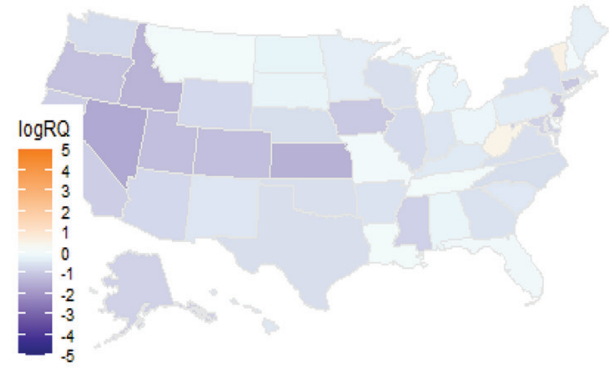

Native Hawaiian or Other Pacific Islander logRQ

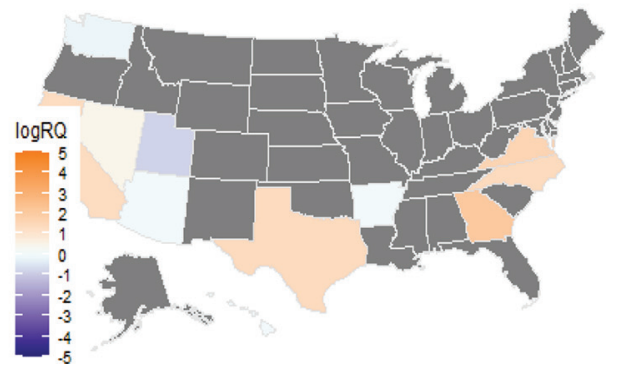

American) and -0.68 (Hispanic or Latino) in 2010s. Asian had been overrepresented since the 1970s, and the $\log R Q$ stabilized at 1.43 during the last 2 decades. White and American Indian or Alaska Native was approximately equitably represented across all 5 decades, with White physicians beginning to be slightly underrepresented in the last 2 decades.

The race/ethnicity proportion and $\log R Q$ of 2020 initial certification candidates relative to 2013 to 2014 AAMC matriculants is presented in Table 4. All racial/ethnic groups had equitable representation, reflected in all $\log \mathrm{RQ}$ s close to 0 , ranging from -0.6 (American Indian or Alaska Native) to 0.3 (Native Hawaiian or Other Islander). Asian, White, Black or
African American, and Hispanic or Latino groups all had $\log$ RQ of 0.1 .

\section{Discussion}

In this study, we utilized the $\log \mathrm{RQ}$ to explore racial/ethnic representation of ABFM certification candidates from the perspective of certification type (initial certification and continuing certification), geographic location (state), historic population change, and educational pipeline. We found representation in Black or African American and Hispanic or Latino increased considerably in the past 4 decades, especially among initial certification candidates. The diversification in ABFM initial 
Table 3. Race/Ethnicity Proportion and $\operatorname{logRQ}$ of Initial Certification Candidates Compared to National Population Race/Ethnicity Proportion by Decades

\begin{tabular}{|c|c|c|c|c|}
\hline Race/Ethnicity & Years & Initial Certification Candidates (\%) & National Population (\%) & $\log \mathrm{RQ}$ \\
\hline \multirow[t]{5}{*}{ White } & 1970 to 1979 & 92.6 & 83.1 & 0.11 \\
\hline & 1980 to 1989 & 91.6 & 80.3 & 0.13 \\
\hline & 1990 to 1999 & 80.9 & 75.1 & 0.07 \\
\hline & 2000 to 2009 & 66.4 & 72.4 & -0.09 \\
\hline & 2010 to $2020^{*}$ & 61.6 & 72 & -0.16 \\
\hline \multirow[t]{5}{*}{ Black or African American } & 1970 to 1979 & 1.4 & 11.7 & -2.12 \\
\hline & 1980 to 1989 & 2.8 & 12.1 & -1.46 \\
\hline & 1990 to 1999 & 4.8 & 12.3 & -0.94 \\
\hline & 2000 to 2009 & 7.1 & 12.6 & -0.57 \\
\hline & 2010 to $2020^{1}$ & 8.1 & 12.8 & -0.46 \\
\hline \multirow[t]{5}{*}{ Asian } & 1970 to 1979 & 4.0 & 1.5 & 0.98 \\
\hline & 1980 to 1989 & 3.5 & 2.9 & 0.19 \\
\hline & 1990 to 1999 & 11.1 & 3.8 & 1.07 \\
\hline & 2000 to 2009 & 20.5 & 4.9 & 1.43 \\
\hline & 2010 to $2020^{*}$ & 23.7 & 5.7 & 1.43 \\
\hline \multirow[t]{5}{*}{ American Indian or Alaska Native } & 1970 to 1979 & 0.7 & 0.6 & 0.15 \\
\hline & 1980 to 1989 & 0.6 & 0.8 & -0.29 \\
\hline & 1990 to 1999 & 0.9 & 0.9 & 0.00 \\
\hline & 2000 to 2009 & 0.9 & 0.9 & 0.00 \\
\hline & 2010 to $2020^{*}$ & 0.9 & 0.9 & 0.00 \\
\hline \multirow[t]{5}{*}{ Hispanic or Latino } & 1970 to 1979 & 2.0 & 6.4 & -1.16 \\
\hline & 1980 to 1989 & 4.2 & 9.0 & -0.76 \\
\hline & 1990 to 1999 & 5.8 & 12.5 & -0.77 \\
\hline & 2000 to 2009 & 7.5 & 12.5 & -0.51 \\
\hline & 2010 to $2020^{*}$ & 9.3 & 18.4 & -0.68 \\
\hline
\end{tabular}

Abbreviation: RQ, representation quotient.

*2019 Census Bureau's American Community Survey (ACS) was used for the population proportion for this decade to be consistent with Table 2 population proportions.

certification candidates is broadly comparable to that in medical school matriculants, indicating that family medicine attracts diverse medical school graduates. Finally, we identified States with a more extreme lack of minority representation in the family medicine workforce.
Younger cohorts of initial certification candidates may be more diverse due to several policies and procedural initiatives enacted over the past few decades, including 1) formal accreditation guidelines that require medical schools to develop programs or partnerships designated to make medical

Table 4. Race/Ethnicity Proportion and $\operatorname{logRQ}$ of 2020 Initial Certification Candidates, Relative to AAMC Matriculants from 2013-2014 (School Year) Race/Ethnicity Proportion

\begin{tabular}{lcrr}
\hline Race & Matriculants (\%) & Initial Certification Candidates (\%) & $\operatorname{logRQ}$ \\
\hline White & 56.9 & 60.1 & 0.1 \\
Black or African American & 7.0 & 7.4 & 0.1 \\
Asian & 20.8 & 23.2 & 0.1 \\
American Indian or Alaska Native & 0.9 & 0.5 & -0.6 \\
Native Hawaiian or Other Islander & 0.3 & 0.4 & 0.3 \\
Hispanic or Latino & 9.1 & 9.3 & 0.1 \\
\hline
\end{tabular}

Abbreviations: AAMC, Association of American Medical Colleges; RQ, representation quotient. 
education more accessible to candidates of diverse backgrounds, including but not limited to sex, race/ ethnicity and socioeconomic status ${ }^{10,15} ; 2$ ) efforts to promote faculty with a diversified background, because they could serve as role models for Underrepresented Minority (URM) students ${ }^{16} ; 3$ ) efforts to enhance college intervention and improvement on URM placement into Graduate Medical Education. ${ }^{17}$ These efforts can be observed in the nearly equitable representation between the 2013 to 2014 medical school matriculants and the 2020 initial certification candidates shown in Table 4 . In addition, although the national population has become more diversified in recent years, the diversification progress has been faster among ABFM initial certification candidates, reflected in the increasing $\log \mathrm{RQ}$ across decades for Black or African American and Hispanic or Latino groups (Table 3). By contrast, older cohorts of continuing certification candidates were less diversified, possibly due to historically racist systems and stringent immigration policies. As the family physician workforce ages and less diverse cohorts retire, the overall workforce will be more diverse and hopefully continue to become more representative of the population it serves. Although there has been progressed in diversification among medical school matriculants, some race/ethnicity groups remain underrepresented; and family medicine must compete with other specialties for the limited number of graduates from underrepresented race/ethnicity groups. This continued underrepresentation of Black or African American, American Indian/Alaskan Native, and Hispanic or Latino is a challenging long-term issue. It is well documented that lack of investment in public education, ${ }^{18}$ disparities in education resource allocation, ${ }^{19}$ as well as the history of school segregation ${ }^{20}$ limit the educational opportunities of these underrepresented minorities, leading to leaks in the pipeline starting with middle school graduates to matriculants of medical schools, and ultimately impacting the physician workforce. Although the governing bodies of medical schools or residency programs could ameliorate some process barriers such as using holistic interviews and multiple mini interviews (MMI), ${ }^{21,22}$ this fundamental pipeline issue must be addressed systematically.

Furthermore, we recognized that Asians are an overrepresented minority group in the health care workforce; however, they are still underrepresented in academic medicine relative to their
White counterparts. ${ }^{11}$ Fassiotto et al found that Asians are underrepresented in US medical school faculty across all faculty ranks, especially in professor rank. ${ }^{11}$ This rank-specific representation approach highlighted the issue of disparities in the medical profession leadership. Aggregate measures of workforce representation might mask the underrepresentation of Asians in leadership roles and hinder the advancement of Asian physicians to higher-level positions.

The state-level analysis reveals geographic variation in levels of representation, calling attention to States that are further behind in attaining a representative family physician workforce. For example, the northwestern States severely lack Black or African American representation even after accounting for the relatively low Black or African American population proportion in the denominator. The same issue applies to American Indians or Alaska Native in the Midwest States and the Native Hawaiian or Other Pacific Islander group who lack representation everywhere except in coastal States. According to AAMC state physician workforce data reports, $40 \%$ of physicians practice in the State where they went to medical school, and $50 \%$ practice in the state of their most recent graduate medical education. Similarly, $56 \%$ of family medicine residency graduates practice within 100 miles of training based on the 2009 American Medical Association Physician Masterfile data. These survey and Masterfile study results suggest that a more widely geographically spread medical school location or resident programs might help build a more diverse physician workforce on the state level. ${ }^{23,24}$

This study has several limitations. First, race and ethnicity are based on self-reported survey data, and candidates are constrained by predetermined socially constructed categories. Individuals of mixed race cannot fully describe the race they identify with because physicians can only choose 1 racial category. The "Other" category could include mixed-race candidates or candidates who do not identify with any existing categories. Second, Asian and Hispanic or Latino are heterogeneous categories, and lumping such diversity might mask other issues of representation. Finally, the $\log R Q$ relative to historic national population data and AAMC medical school data are all based on common categories; when uncommon categories such as 2 or more races are offered, strictly direct comparisons become impossible. 


\section{Conclusion}

We utilized the proposed $\log \mathrm{RQ}$ metric to quantify the advancement in representation among ABFM certification candidates in various contexts. We found that while representation of Black or African American and Hispanic or Latino family physicians increased substantially in the past 2 decades, those groups remain underrepresented. At the state level, we found variation in representation for certain racial/ethnic groups. These findings raise the concern that there are not enough physicians from certain racial/ethnic groups to provide adequate patient-physician concordant health care; however, as a specialty, we found that family medicine attracts representative medical school matriculants. If the medical educational pipeline continues to diversify, the family medicine workforce will become more diverse and representative as well. The proposed $\log \mathrm{RQ}$ may serve as a useful tool to monitor representation progress or identify representation disparity for specific demographic groups on various levels to ensure continued efforts to diversification in the family physician workforce.

To see this article online, please go to: http://jabfm.org/content/ 35/1/9.full.

\section{References}

1. Shen MJ, Peterson EB, Costas-Muñiz R, et al. The effects of race and racial concordance on patient-physician communication: a systematic review of the literature. J Racial Ethn Health Disparities 2018;5:117-40.

2. Greenwood BN, Hardeman RR, Huang L, Sojourner A. Physician-patient racial concordance and disparities in birthing mortality for newborns. Proc Natl Acad Sci 2020;117:21194-200.

3. Jetty A, Jabbarpour Y, Pollack J, Huerto R, Woo S, Petterson S. Patient-Physician Racial Concordance Associated with Improved Healthcare Use and Lower Healthcare Expenditures in Minority Populations. J Racial Ethn Health Disparities 2021.

4. Saha S, Taggart SH, Komaromy M, Bindman AB. Do Patients Choose Physicians Of Their Own Race?: To provide the kind of care consumers want, medical schools might be able to justify using race as an admissions criterion. Health Aff (Millwood) 2000;19:76-83.

5. LaVeist TA, Nuru-Jeter A. Is doctor-patient race concordance associated with greater satisfaction with care? J Health Soc Behav 2002;43:296-306.

6. Alsan M, Garrick O, Graziani G. Does Diversity Matter for Health? Experimental Evidence from Oakland. American Economic Review 2019;109: 4071-111.071-4111.
7. Petersdorf RG, Turner KS, Nickens HW, Ready T. Minorities in medicine: past, present, and future. Acad Med 1990;65:663-70.

8. Xierali IM, Nivet MA. The Racial and Ethnic Composition and Distribution of Primary Care Physicians. J Health Care Poor Underserved 2018; 29:556-70. doi:10.1353/hpu.2018.0036

9. Peabody MR, Eden AR, Douglas M, Phillips RL. Board Certified Family Physician Workforce: Progress in Racial and Ethnic Diversity. J Am Board Fam Med 2018;31:842-3.

10. Lett LA, Murdock HM, Orji WU, Aysola J, Sebro R. Trends in Racial/Ethnic Representation Among US Medical Students. JAMA Netw Open 2019;2:e1910490.

11. Fassiotto M, Flores B, Victor R, et al. Rank Equity Index: Measuring Parity in the Advancement of Underrepresented Populations in Academic Medicine. Acad Med 2020;95:1844-52.

12. Anderson CJ, Verkuilen J, Johnson T. Applied Generalized Linear Mixed Models: Continuous and Discrete Data. Springer; 2012. Available at: https:// education.illinois.edu/docs/default-source/carolynanderson/edpsy587/GLM_GLMM_LMM.pdf. Accessed October 4, 2021.

13. Wikipedia Website. Available at: https://en.wikipedia. org/wiki/Historical_racial_and_ethnic_demographics_ of_the_United_States. Accessed October 8, 2021.

14. AAMC Website. Available at: https://www.aamc. org/data-reports/students-residents/interactivedata/facts-glossary. Accessed October 8, 2021.

15. Boatright DH, Samuels EA, Cramer L, et al. Association between the Liaison Committee on Medical Education's diversity standards and changes in percentage of medical student sex, race, and ethnicity. Jama 2018;320:2267-9.

16. Daley S, Wingard DL, Reznik V. Improving the retention of underrepresented minority faculty in academic medicine. J Natl Med Assoc 2006;98: 1435-40.

17. Sondheimer HM, Xierali IM, Young GH, Nivet MA. Placement of US medical school graduates into graduate medical education, 2005 through 2015. JAMA 2015;314:2409-10.

18. Flores RL. The rising gap between rich and poor: A look at the persistence of educational disparities in the United States and why we should worry. Cogent Social Sciences 2017;3:1323698.

19. Jackson D. Why am I behind? an examination of low income and minority students' preparedness for college. McNair Sch J 2012;13:121-38.

20. Rothstein R. The racial achievement gap, segregated schools, and segregated neighborhoods: A constitutional insult. Race Soc Probl 2015;7:21-30.

21. Prober CG, Kolars JC, First LR, Melnick DE. A plea to reassess the role of United States Medical Licensing Examination Step 1 scores in residency selection. Acad Med 2016;91:12-5. 
22. Sherman M, Ricco J, Nelson S, Nezhad S, Prasad S. Implicit bias training in a residency program: aiming for enduring effects. Fam Med 2019;51: 677-81.

23. Association of American Medical Colleges. 2017 state physician workforce data report. Available at: https://store.aamc.org/2017-state-physician-workforcedata-report.html. Accessed May 14, 2021.

24. Fagan EB, Finnegan SC, Bazemore A, Gibbons C, Petterson S. Migration after family medicine residency: $56 \%$ of graduates practice within 100 miles of training. Am Fam Physician 2013;88:704. 